\title{
Aliança da enfermagem com o usuário na defesa do SUS
}

\author{
Nursing partnership with users in the defense of SUS \\ Alianza de la enfermería con el usuario en la defensa del SUS
}

\section{Carla Aparecida Arena Ventura', Débora Falleiros de Mello", Raquel Dully Andrade"II, Isabel Amélia Costa Mendes ${ }^{\text {IV }}$}

\author{
' Universidade de São Paulo, Escola de Enfermagem de Ribeirão Preto, \\ Departamento de Enfermagem Psiquiátrica e Ciências Humanas. Ribeirão Preto-SP, Brasil. \\ "Universidade de São Paulo, Escola de Enfermagem de Ribeirão Preto, \\ Departamento de Enfermagem Materno Infantil e Saúde Pública. Ribeirão Preto-SP, Brasil. \\ III Universidade Estadual de Minas Gerais, Curso de Enfermagem. Passos-MG, Brasil. \\ "v Universidade de São Paulo, Escola de Enfermagem de Ribeirão Preto, \\ Departamento de Enfermagem Geral e Especializada. Ribeirão Preto-SP, Brasil.
}

\author{
Submissão: 20-12-2012 Aprovação: 20-12-2012
}

\section{RESUMO}

Estudo reflexivo com o objetivo de apresentar, na perspectiva dos direitos humanos, elementos relevantes para o cuidado de enfermagem na defesa dos sujeitos no contexto do Sistema Único de Saúde (SUS) brasileiro. A saúde como um direito de todos e um dever do Estado materializou-se como uma conquista da população com o SUS, com os princípios de universalidade, integralidade e equidade da atenção em saúde, ampliando a concepção de saúde para além da ausência de doença e passando a ser entendida como qualidade de vida. O enfermeiro tem grande potencial para desempenhar um papel singular na defesa do direito à saúde e do direito à vida e, por meio de alianças, fortalecer o exercício da cidadania das pessoas.

Descritores: Enfermagem; Direito à Saúde; Integralidade.

\section{ABSTRACT}

This reflexive study aimed at presenting, based on the human rights framework, relevant elements for nursing care in the defense of the users of the Brazilian Single Health System (SUS). The assurance of health as a fundamental right and a government's responsibility was an achievement of the population through SUS, founded on the principles of universal, integral and equitable care, expanding the conception of health beyond the idea of the lack of disease, understood as quality of life. Nurses have a potential to perform a single role in the defense of the right to health and right to life and, by means of different partnerships, to strengthen the exercise of citizenship.

Key words: Nursing; Right to Health; Integrality.

\section{RESUMEN}

Estudio reflexivo con el objetivo de presentar, en la perspectiva de los derechos humanos, elementos relevantes para el cuidado de enfermería en la defensa de los sujetos en el contexto del Sistema Único de Salud (SUS) brasileño. La salud como un derecho de todos y un deber del Estado se ha consolidado como una conquista de la población con él SUS, con los principios de universalidad, integralidad y equidad de atención en salud, ampliando la concepción de salud para además de ausencia de enfermedades y que pasó a ser comprendida como calidad de vida. El enfermero tiene grande potencial para desarrollar un rol singular en la defensa del derecho a salud y del derecho a vida y, por medio de alianzas, fortalecer el ejercicio de la ciudadanía de las personas.

Palabras clave: Enfermería; Derecho a Salud; Integralidad.

Conferência de encerramento do $64^{\circ}$ Congresso Brasileiro de Enfermagem, promovido pela ABEn Nacional e realizado pela $A B E n$ Seção Rio Grande do Sul, no período de 29/10 a 1/11 de 2012, em Porto Alegre-RS, Brasil.

\section{AUTOR CORRESPONDENTE Carla Aparecida Arena Ventura E-mail: caaventu@gmail.com}




\section{INTRODUÇÃO}

O Sistema Único de Saúde (SUS) representa uma grande conquista, pois materializa, em sua organização física e estruturação, a possibilidade do exercício do direito humano à saúde. Contudo, as experiências das pessoas que vivenciam o cuidado cotidiano no contexto dos serviços públicos de saúde brasileiros nem sempre são positivas, o que requer uma aproximação maior com relação aos sujeitos e o modo de organização dos serviços de saúde, buscando-se compreender melhor a baixa resolutividade de alguns serviços, casos de deterioração da qualidade do cuidado e, principalmente, de violação de direitos humanos.

Diante de tantas incongruências e desigualdades no exercício de nossos direitos, nos indagamos cada vez mais: será que todos nós temos os mesmos direitos? Afinal, o que significa ter direitos? O que é direito?

Nessa perspectiva, muitas vezes não nos damos conta de que somos titulares de direitos, de que o direito é um processo de construção social e que vai muito além da existência de leis e do monopólio estatal.

As dificuldades na compreensão de nossos direitos foram evidenciadas em uma pesquisa de opinião pública realizada pela Secretaria de Direitos Humanos da Presidência da República publicada em 2010, que apresentou como objetivo conhecer o que são os direitos humanos para os cidadãos brasileiros. Como resultados, dentre os direitos humanos primeiramente elencados pelos entrevistados estão os direitos sociais $(68 \%)$, ou seja, os que dependem totalmente do Estado para a sua concretização, com destaque o direito à saúde (com $47 \%)$, à educação $(38 \%)$ e ao trabalho $(26 \%)^{(1)}$. Os achados demonstraram, ainda, que o conceito de cidadania remete os brasileiros a noções abrangentes e universalistas e aos direitos sociais. Nesse sentido, é importante ressaltar que os entrevistados não mencionaram a importância dos direitos políticos para o exercício da cidadania, referindo-se à família, que é essencialmente um espaço privado, como o local privilegiado para o aprendizado dos direitos humanos. Verifica-se aqui um paradoxo nessas visões, pois o exercício da cidadania não faz sentido fora de uma referência coletiva e os resultados da pesquisa associaram o exercício de direitos eminentemente à esfera privada ${ }^{(1)}$. Os resultados demonstraram também que um em cada sete brasileiros entende os direitos como privilégios de grupos restritos da população e dois em cada cinco brasileiros não sabem mencionar sequer um direito humano assegurado na Constituição ${ }^{(1-2)}$.

Podemos inferir, então, que quanto menos o Estado pode garantir aos cidadãos a segurança pessoal mínima, mais existe a carência de direitos como saúde, educação, moradia, o que obscurece a dimensão política da vida, no sentido amplo da palavra. Quando não há direitos políticos, os outros direitos são concedidos, apenas, como favor ou privilégio ${ }^{(2)}$. As pessoas condenadas a viver no reino da necessidade são ao mesmo tempo as que mais carecem de cidadania e as que possuem menos condições de conquistá-la por seu próprio esforço ${ }^{(3)}$.

Nessa perspectiva, é importante destacar que, seja com caráter de recomendação, como a Declaração Universal dos
Direitos Humanos de 1948, ou a Constituição brasileira, com força legal, nenhum instrumento sozinho tem como garantir a plena vigência e universalização de direitos neles reconhecidos. Sendo assim, é fundamental a nossa participação no processo de consolidação dos direitos. O direito não tem a capacidade de alterar imediatamente a realidade, mas constitui um instrumento a ser utilizado por nós para tal. Ainda, está em constante processo de construção e reconstrução pela própria sociedade ${ }^{(4)}$.

Este artigo baseia-se, portanto, na concepção de um direito plural, que está presente em diferentes espaços de sociabilidade. Fundamenta-se no imperativo de que o aprendizado pode reduzir a chance de violações aos direitos das pessoas.

Considerando que as práticas de saúde necessitam ser fortalecidas com a atuação dos profissionais e dos sujeitos na construção de planos de responsabilização, em busca da integralidade da atenção e do exercício do direito à saúde, nesta reflexão discutem-se as possibilidades de atuação dos enfermeiros em sua interação com a sociedade, com base nos princípios dos direitos humanos. Exploram-se, a seguir, algumas potencialidades da aliança da Enfermagem com os usuários na defesa do SUS.

\section{O DIREITO HUMANO À SAÚDE E OS DIREITOS DOS USUÁRIOS DO SISTEMA ÚNICO DE SAÚDE}

O conceito de saúde carrega em si um longo processo de discussão e negociação que vem ocorrendo em sintonia com o que se entende por cidadania ${ }^{(5)}$. O direito à saúde foi proclamado no art. 25 da Declaração Universal dos Direitos Humanos em 1948 e, em 1966, confirmado pelo Pacto da Organização das Nações Unidas (ONU) sobre Direitos Econômicos, Sociais e Culturais. Ainda, desde 1978, com a Primeira Conferência Internacional sobre Cuidados Primários de Saúde realizada em Alma Ata, a temática dos direitos humanos tornou-se um eixo para as discussões sobre as políticas de saúde. A partir deste evento, internacionalmente chegou-se ao consenso de que a saúde constitui um direito humano fundamental e que requer a participação da sociedade no planejamento e na prestação de serviços e cuidados. No Brasil, apesar de todos esses avanços, a saúde somente foi garantida como direito fundamental a toda a população por meio do art. 196 da Constituição de $1988^{(6)}$.

O conceito de saúde possui implicações legais, sociais e econômicas. Sendo assim, o contexto de desigualdades do exercício do direito à saúde pela sociedade, causando uma real exclusão social em saúde, ainda é vivenciado por grande parte da população. Dessa forma, discutimos o conceito de saúde com base em elementos sociais, econômicos e políticos, mas, acima de tudo, como um direito humano fundamental. Sendo assim, iniquidades, pobreza, exploração, violência e injustiça social são causas da deterioração das condições de saúde de grande parte da população pobre e marginalizada, que está totalmente excluída das esferas de poder; pessoas que são moldadas pelo contexto em que vivem e que não possuem a chance de interferir neste contexto; pessoas que historicamente foram usadas como "meios" e nunca como "fins" 
neste processo, pessoas que não são realmente consideradas sujeitos de direitos ${ }^{(6-7)}$.

Nesse sentido, a criação do SUS representa uma grande conquista, pois se afirma na busca de uma nova ordem social focada na promoção do bem estar de todas as pessoas. Nessa perspectiva, espera-se que o agir em saúde seja democrático, com respeito, responsabilidade e solidariedade, de maneira transformadora, humanizada e emancipadora, reconhecendo-se a integralidade do direito à saúde no país.

Em compasso com os princípios de uma nova ordem social, valorizando a saúde como um direito de todos e dever do Estado, e considerando a Lei 8.080 de 1990 que dispõe sobre as condições para a promoção, proteção e recuperação da saúde, a organização e funcionamento dos serviços correspondentes e a Lei 8.142 também de 1990 que estabelece a participação da comunidade na gestão do SUS e dispõe sobre as transferências intergovernamentais de recursos financeiros na área da saúde, o próprio Sistema Saúde elenca como direitos dos usuários do SUS:

1. Todo cidadão tem direito ao acesso ordenado e organizado aos sistemas de saúde;

2. Todo cidadão tem direito a tratamento adequado e efetivo para seu problema;

3. Todo cidadão tem direito ao atendimento humanizado, acolhedor e livre de qualquer discriminação;

4. Todo cidadão tem direito a atendimento que respeite a sua pessoa, seus valores e seus direitos;

5. Todo cidadão também tem responsabilidades para que seu tratamento aconteça da forma adequada;

6. Todo cidadão tem direito ao comprometimento dos gestores da saúde para que os princípios anteriores sejam cumpridos.

Todavia e como já mencionado anteriormente, não basta à legislação para que ocorram transformações. Dessa forma, tais princípios somente serão uma realidade se ocorrerem mudanças de atitudes nas práticas de atenção e gestão, buscando-se fortalecer a autonomia e o direito do cidadão. Ainda, é importante considerar que esse processo de transformação é bastante complexo e que as atitudes das pessoas com relação à saúde e ao direito à saúde são influenciadas por fatores em diferentes níveis ${ }^{(6)}$, ilustrados sucintamente na Figura 1.

Visando fomentar atitudes mais pró-ativas dos usuários com relação aos serviços de saúde, é necessário aumentar o vínculo com a população, a adesão às medidas de proteção e promoção da saúde e, principalmente, potencializar a atuação dos profissionais de saúde junto às famílias, bem como a construção de projetos de saúde com a responsabilização dos sujeitos envolvidos.

Nesse cenário, qual pode ser o papel desempenhado pela Enfermagem? Há realmente uma aliança entre a enfermagem e os usuários do SUS em sua defesa? Ainda, qual é o significado da busca desta defesa? Por que a Enfermagem pode e deve liderar este processo? O que faz com que a Enfermagem faça a diferença na relação com os usuários do SUS e na promoção de seus direitos, especialmente de seu direito à saúde?

\section{A ENFERMAGEM E OS USUÁRIOS DOS SUS}

Podemos afirmar, com base na compreensão do cuidado em saúde como uma categoria reconstrutiva, que envolve voltar-se à presença do outro, otimizar a interação, procurando ter presença ativa, redes de conversação e expansão de horizontes $^{(8)}$, que o enfermeiro desempenha um papel como agente político, de cidadania, o que não compromete sua atuação técnica. O papel político desenvolvido pelos enfermeiros junto aos usuários pode ser denominado como advocacia.

Sendo assim, a advocacia representa um dos conceitos chave da ética em enfermagem, discutido na literatura desde 1973 quando o Conselho Internacional de Enfermeiros (CIE) o introduziu no Código de Ética da profissão. Dessa forma, de acordo com o CIE, o enfermeiro possui o papel inerente à profissão de respeito aos direitos humanos, incluindo o direito à vida, à dignidade e de ser tratado com respeito.

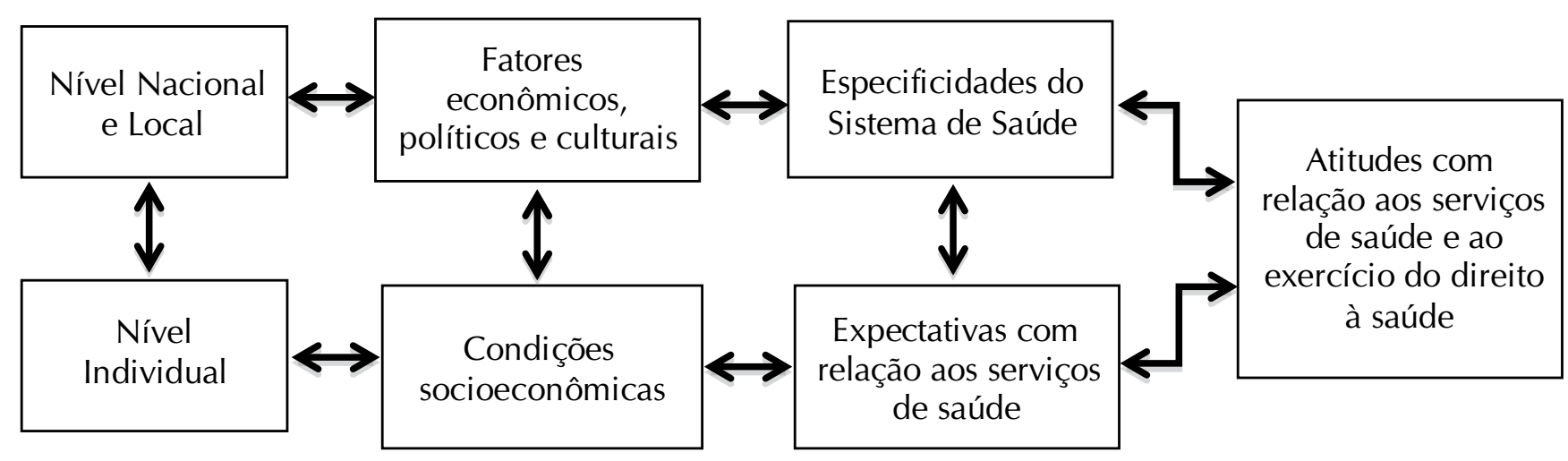

Figura 1 - Contexto nacional e local (fatores econômicos, políticos e culturais), o sistema de saúde e elementos individuais (condições socioeconômicas e expectativas com relação aos serviços de saúde). 
A ideia de fortalecimento da atuação do enfermeiro na advocacia em saúde é recorrente em diversas organizações nacionais e internacionais ligadas à profissão. A própria Rede Global de Centros Colaboradores da Organização Mundial da Saúde (OMS) para o Desenvolvimento da Enfermagem e Obstetrícia, formada por 44 Centros Colaboradores provenientes das seis regiões de trabalho da OMS, reafirma que sua missão é otimizar o papel de advocacia dos enfermeiros (nos serviços, mas também nas instituições acadêmicas), maximizando sua contribuição para aperfeiçoar as ações de saúde dos Estados membros e de outras organizações para a promoção da saúde das pessoas.

Verifica-se, então, o quão importante é esta conscientização do enfermeiro sobre o seu papel junto aos usuários dos serviços de saúde, em diferentes esferas de sua atuação. Não buscamos aqui minimizar a relevância das ações técnicas específicas da enfermagem. Além da excelência na realização de suas atividades, o enfermeiro desempenha, em sua prática, um contato direto com situações que o levam a agir também como agente político em busca de mudanças, como "advogado" dos usuários dos serviços de saúde.

Os enfermeiros são profissionais diferenciados, devido aos seus conhecimentos técnicos, habilidades holísticas e a possibilidade de advogarem pelos usuários dos serviços de saúde, reconhecendo também a atuação de outros profissionais da área. Dessa forma, a advocacia em saúde pode e também é desempenhada pelos profissionais de saúde na defesa de diversos aspectos do processo de cuidar em saúde, destacando-se o enfermeiro como um profissional-chave e responsável pelas ações de acompanhamento dos usuários do Sistema Único de Saúde.

Em conjunto com os usuários, os enfermeiros podem auxiliar no processo de empoderamento dos sujeitos, para que se tornem usuários ativos e corresponsáveis pelo cuidado. $\mathrm{O}$ conceito de advocacia vem do latim "advocatus" que significa aquele que oferece evidências ${ }^{(9)}$. As ações de advocacia são definidas como apoio verbal ou argumentação com relação a uma causa, como função do advogado.

De acordo com a OMS, as ações de advocacia incluem as iniciativas de equipes técnicas de fornecer evidências às instituições e organizações acerca de um desafio importante para a saúde, assim como de distribuir informações entre diferentes canais, visando chegar ao público em geral, ou ações para aumentar a conscientização sobre um problema específico. Engloba ações de defesa de direitos e são aplicáveis especialmente às pessoas cujos direitos e necessidades são ignorados, às pessoas que são, algumas vezes, invisíveis em nossa sociedade. O conceito de advocacia compreende um processo que visa mobilizar apoio para uma causa, buscando provocar mudanças favoráveis, por meio da formação de parcerias com indivíduos ou grupos que possuem objetivos comuns ${ }^{(10)}$.

A advocacia em saúde é um processo que utiliza estratégias políticas com o objetivo de promover direitos não respeitados, procurando influenciar particulares e autoridades, sensibilizando-os para carências e necessidades diversas, frente a alguma forma de vulnerabilidade vivenciada pelo sujeito assistido.
Para enfrentar com os usuários esta condição de vulnerabilidade, é fundamental investir em ações de educação em saúde, que também configuram parte das atividades do enfermeiro no exercício da advocacia em saúde. Os direitos dos usuários podem ser promovidos à medida que conheçam quais são os seus direitos, que estejam em posição para pesar diferentes opções de cuidado e realmente possam tomar uma decisão informada sobre sua própria saúde. Nesse contexto, a advocacia do enfermeiro deve ser vista como parte integral em sua atuação profissional para a promoção do bem estar do usuário do serviço de saúde, assim como na construção de sua autonomia. Famílias capacitadas e conscientes terão autonomia para realizar modificações no ambiente e em suas relações, adotando comportamentos favoráveis e buscando a efetivação do direito à saúde $\mathrm{e}^{(11-12)}$.

Este aspecto da advocacia foi incorporado no Código de Enfermagem de muitos países. Nessa perspectiva, proteger os usuários dos serviços de saúde contra a incompetência de outros profissionais de saúde faz também parte do papel de advocacia do enfermeiro.

Para ilustrar a concepção do enfermeiro como agente na advocacia em saúde, a partir de revisão da literatura sobre advocacia em enfermagem ${ }^{(13)}$, são apontadas três definições comuns para esse processo: 1. a advocacia é motivada pelos direitos do usuário à informação e autodeterminação; 2. a advocacia derivada do direito do usuário à segurança pessoal e 3. a advocacia como um princípio filosófico da enfermagem.

A definição da advocacia com base nos direitos individuais à informação e autodeterminação aparece em vários estudos. Este conceito pró-ativo da advocacia inclui ações que buscam auxiliar o usuário a definir seu desejo de modo que possa realmente tomar uma decisão informada sobre o seu próprio cuidado. Em contraposição à primeira definição, a advocacia ligada aos direitos do usuário à segurança pessoal é reativa e necessária em situações em que os pacientes estão em perigo. Ainda, a advocacia como um princípio filosófico em enfermagem, relaciona-se às competências de enfermagem, especialmente ligadas ao reconhecimento da dimensão moral de alguns problemas e aos dilemas éticos enfrentados pelos enfermeiros no cuidado dos usuários dos serviços de saúde ${ }^{(13-14)}$.

Como resultado das ações de advocacia realizadas pelo enfermeiro, a maioria dos trabalhos aponta, com relação ao usuário, a possibilidade de seu empoderamento e promoção de sua autodeterminação. No que diz respeito ao próprio enfermeiro, potencialização de sua autonomia e empoderamento. De forma geral, as consequências dessa aliança são consideradas positivas para ambas as partes (usuários e enfermeiros), bem como para a sociedade ${ }^{(13-14)}$.

Contudo, não podemos deixar de mencionar que este processo pode não ser fácil, principalmente para os enfermeiros. Muitas vezes, se sentem isolados e não contam com a interação e colaboração de outros profissionais de saúde. Apesar das adversidades vislumbradas na prática, muitos enfermeiros buscam soluções e são bastante criativos para exercerem também a sua função política, como advogados dos usuários dos serviços de saúde, pois possuem uma perspectiva única no contexto dos serviços de saúde. Ao estarem mais próximos 
dos usuários, têm a oportunidade para conhecê-los melhor, estabelecer uma relação terapêutica e atuar como mediadores em diferentes situações entre os próprios usuários e os outros profissionais de saúde. São também os que conseguem detectar mais facilmente os erros, tendo oportunidades singulares para preveni-los. Sendo assim, os enfermeiros protegem os usuários e também as organizações das quais fazem parte, por meio da advocacia, à medida que identificam e analisam questões individuais e organizacionais.

Como resposta, as organizações, quando ouvem os seus enfermeiros, podem resolver problemas que potencialmente afetam a segurança dos usuários dos seus serviços. Para tanto, é preciso que os enfermeiros se sintam confiantes o suficiente para exporem as situações e atuarem na liderança destas mudanças, adotando diferentes estratégias de comunicação ${ }^{(15)}$ com os seus superiores e outros profissionais da equipe de saúde, enfrentando conflitos e buscando ser pró-ativos.

Nesse cenário, como líderes da equipe de enfermagem, é fundamental que os enfermeiros promovam uma cultura de valorização da comunicação, de abertura a críticas e de construção de contínua capacitação. As razões atribuídas por enfermeiros para não relatarem situações de risco são o medo de retaliação, a crença de que falar não é comum e de que ninguém faz isto (se ninguém faz, porque eu vou fazer?). Em algumas organizações, o ato de questionar é considerado como de alto risco e baixo benefício ${ }^{(13-14)}$. Tradicionalmente, temos uma cultura de negar a existência de conflitos, de postergar a solução de situações difíceis e de não enfrentamento, o que faz com que os problemas cresçam cada vez mais. Deve-se, portanto, buscar fazer o oposto, passar a ter consciência das limitações, lidando com elas e acreditando que ao enfrentarmos abertamente os conflitos, iremos aprender com este processo.

É relevante ressaltar, ainda, a advocacia coletiva, especialmente no âmbito político, que ocorre mais efetivamente por meio de organizações profissionais. A Associação Brasileira de Enfermagem constitui um excelente exemplo de advocacia da enfermagem. A advocacia no nível macro se desenvolve de forma efetiva por meio das associações profissionais, que podem promover mudanças políticas e apoiar esforços individuais de advocacia.

A força da advocacia em saúde pelo enfermeiro pode ser vista como um símbolo de unidade de propósito e representa um posicionamento de vida. Algumas vezes, o enfermeiro pode achar que advogar pelo usuário é chamar o médico, ou pedir uma medicação e esta limitada interpretação reduz a importância deste papel. Em contraposição, enfatizamos que o enfermeiro pode e deve assumir o papel maior que o de um mediador. Deve buscar assegurar os direitos dos usuários, especialmente o direito a um cuidado integral e de qualidade, que preserve seus valores na tomada de decisão, garantindo sua autodeterminação. Sendo assim, o objetivo final é empoderar o usuário do serviço de saúde, construindo com ele significados para suas experiências.

\section{CONSIDERAÇÕES FINAIS}

Além do êxito técnico do enfermeiro direcionado ao caráter instrumental de suas ações específicas, ao alcance de determinados resultados e a atenção aos agravos à saúde de indivíduos e populações, o enfermeiro busca o sucesso prático, preocupando-se com o sentido que as ações de saúde têm para os sujeitos e para as populações. Este é um exercício real de cidadania: o enfermeiro passa a se considerar sujeito de direitos e permite, por meio de suas ações e do respeito ao outro também como sujeito de direitos, que o usuário dos serviços de saúde também exerça sua cidadania.

A enfermagem possui uma perspectiva ímpar e pode liderar este processo de ação em meio a tantas dificuldades e desigualdades, em um contexto que por alguns pode ser considerado impossível. O enfermeiro tem grande potencial para desempenhar um papel singular na defesa do direito à saúde e do direito à vida e, por meio de alianças, fortalecer o exercício da cidadania das pessoas.

\section{REFERÊNCIAS}

1. Venturi G. O potencial emancipatório e a irreversibilidade dos direitos humanos. In: Venturi G, editor. Direitos humanos: percepções da opinião pública. Brasília: Secretaria de Direitos Humanos da Presidência da República; 2010. p. 9-32.

2. Kehl MR. Direitos humanos: a melhor tradição da modernidade. In: Venturi G, editor. Direitos humanos: percepções da opinião pública. Brasília: Secretaria de Direitos Humanos da Presidência da República; 2010. p. 33-41.

3. Arendt H. A condição humana. Rio de Janeiro: Forense Universitária; 2010.

4. Arendt H. Origens do totalitarismo. São Paulo: Companhia das Letras; 2011.
5. Lopes F, Magalhães AF. Saúde, direitos humanos e cidadania no Brasil. In: Venturi G, editor. Direitos humanos: percepções da opinião pública. Brasília: Secretaria de Direitos Humanos da Presidência da República; 2010. p. 209-17.

6. Ventura CAA. Saúde mental e direitos humanos: o processo de construção da cidadania de pessoas portadoras de transtornos mentais. Ribeirão Preto. Tese [Livre Docência em Enfermagem]- Universidade de São Paulo, 2011.

7. Schrecker T, Chapman AR, Labonté R, De Vogli R. Advancing health equity in the global marketplace: how human rights can help. Soc Sci Med 2010;71(8):1520-6.

8. Ayres JRCM. Cuidado: trabalho e interação nas práticas de saúde. Rio de Janeiro: CEPESC; IMS-UERJ; ABRASCO; 2009. 
9. Milner $\mathrm{N}$. The symbols and meanings of advocacy. Int J Law Psychiatry 1986;8(1):1-17.

10. World Health Organization. Advocacy: a practical guide with polio eradication as a case study. Geneva: World Health Organization; 1999.

11. Grippo MLVS, Fracolli L. A. Avaliação de uma cartilha educativa de promoção ao cuidado da criança a partir da percepção da família sobre temas de saúde e cidadania. Rev Esc Enferm USP 2008;42(3):430-6.

12. Andrade RD, Mello DF, Silva MAI, Ventura CAA.
Advocacia em saúde na atenção à criança: revisão da literatura. Rev Bras Enferm 2011;64(4):738-44.

13. Vaartio $H$, Leino-Kilpi $H$. Nursing advocacy: a review of the empirical research 1990-2003. Int J Nurs Stud 2005;42(6):705-14.

14. Zolnierek CD. Beyond rhetoric: supporting patient advocacy in nursing practice. Perioper Nurs Clin 2012;7(4):379-87.

15. Bermejo JC. Salir de la noche: por una enfermería humanizada. Serie: Humanizar la salud. Santander: Editorial Salterrae; 1999. 